\title{
Research on the Lysozyme imprinted polymers BaiyiXie ${ }^{1, a}$, Aiqin Luo, a
}

\author{
Department of life science, Beijing Institute of Technology,Beijing, 100081, China \\ email:aqluobit@163.com,
}

Corresponding Author: Aiqin Luo

Keywords: Lysozyme, Imprinted polymers, Hydrogels

\begin{abstract}
Acrylamide as functional monomer, N, N'- methylene-bis-acrylamide as crosslinking agent, prepared lysozyme (Lyz) molecularly imprinted polymer hydrogel and UV spectrophotometer Adsorption of polymersIt was characterized. The results showed that polyacrylamide hydrogel successfully formed a lysozyme molecularly imprinted; good adsorption kinetics and thermodynamics properties of imprinted and non-imprinted polymer; the amount of imprinted polymer adsorption is significantly greater than the amount of non-imprinted polymer..
\end{abstract}

\section{Introduction}

Molecular imprinting technique (Molecular Imprinting Technique, MIT) has developed rapidly since the 1970s. Especially after Mosbach et al publishedan article in "Nature" on the theophylline imprinted polymer ${ }^{[1]}$ (Molecular Imprinted Polymers, MIPs), the techniquedeveloped very rapidly since then. This is mainly because thatthe Molecular Imprinting Techniquehas three characteristics: the identification absorption, predetermined structural and real practicality ${ }^{[2]}$. MIPs tolerance to extreme environments is very strong, and has a long service life. MIPs binds with template molecule, thenfinishes elution to remove the template molecule, thus forming a molecularly imprinted polymerswhich have highly specific binding regions ${ }^{[3]}$.

In proteins as separate objects, as a template molecules synthesized molecularly imprinted polymers to prepare imprinted polymer technology called protein molecular imprinting technique. This technology has good physical and chemical stability, can withstand high temperature, acid and organic solvents, long life, high specificity, good separation, inexpensive, process reproducibility number, suitable for mass production ${ }^{[4]}$ 。

In this work, choosing the Lysozyme as the template molecule, acrylamide as functional monomerandN, N'- methylene-bis-acrylamide as crosslinking agent,using the bulk polymerization method and forming the Lysozyme imprinted polymers.

\section{Experimental}

\section{Materials}

Lysozyme (Lyz, pI 11.2,MW 14.4 kDa), ovalbumin(OVA, pI 4.7, MW 43.0 kDa), and bovine serum albumin (BSA, pI 4.9, MW 66.0 kDa) were obtained from Hess Leber Biotechnology Co. Ltd.Beijing. Acrylamide(AAm) and N, N'- methylene-bis-acrylamide(Mba) were obtained from Tianjin Guangfu Fine Chemical Research Institute.

\section{Synthesis of Lysozyme imprinted polymers}

Lyz (50mg) ,AAm (480mg), Mba(12mg) were dissolved in PBS(pH7.0, 20mM) .Keeping the solution in ice-bath for pre-polymerization in one hour. Then adding APS $(2 \mathrm{ul}, 100 \mathrm{mg} / \mathrm{mL})$ and TEMED ( $10 \mathrm{ul}, 30 \mathrm{vol} \%)$ in the sequence. After every reagents have fully dissolved in the solution, putting the solution into the water-bath at the 30 degrees centigrade for 24 hours under the $\mathrm{N}_{2}$ circumstance. The resultant clear gel products were washed with D.D water and put in vacuum for further using.

Crashed the polymers and made them into the eluent (10vol\%,10wt $\%$ ) under oscillating for one 
hour. Repeating the step six times and then using the D.D water doing the same action times until the $\mathrm{pH}$ of the supernatant being 7.0.

\section{Binding experiment}

\section{Binding kinetics}

Binding kinetics was tested by changing the adsorption time at regular intervals from 1 to 5 hours with the same initial concentration of $\operatorname{Lyz}(0.25 \mathrm{mg} / \mathrm{ml})$. The adsorption capacity(Q) of the template molecule or competitive molecule bound to the polymers is defined as

$$
\mathrm{Q}=\frac{\left(C_{e}-C_{0}\right) \mathrm{V}}{M}
$$

$\mathrm{C}_{0}$ and $\mathrm{Ce}$ are the initial concentration and the supernatant concentration of the template molecule, $\mathrm{V}$ is the volume of the initial solution, and $\mathrm{W}$ is the weight of the imprinted polymers.

\section{Binding isotherms}

Binding isotherms were achieved by batch binding tests, experiment was tested by changing the concentrations of Lyz from 0.05 to $1.0 \mathrm{mg} / \mathrm{mL}$ while keeping the same adsorption time of 4 hours. And the binding capacity was calculated with the same method.

\section{Competitive binding test}

Competitive binding tests were verified by using the mixed solution. $10 \mathrm{mg}$ MIPs or NIPs were subjected to the ternary protein mixture solution of BSA $(0.5 \mathrm{mg} / \mathrm{mL})$, OVA $(0.5 \mathrm{mg} / \mathrm{mL})$ and Lyz $(0.5 \mathrm{mg} / \mathrm{mL})$. Theadsorption experiments were carried out in a same manner as mentioned above. The concentration of each protein in the mixture solutions was determined using HPLC method.

The concentration of single protein solution was obtained by using a UV-1800 spectrophotometer (Shimadzu, Japan). HPLC analysis of protein samples was performed on Waters ACQUITY UPLC H-Class system (USA) with a Tosoh TSKgel C4-300 (150 mm $94.6 \mathrm{~mm}, 3 \mathrm{~lm}$, 300 A ) column (Japan). Gradient elution program was as follows: solution A: 10 vol\%acetonitrile and 90 vol\% D.I. water with 0.05 vol\% trifluoroacetic acid (TFA), solution B: $80 \mathrm{vol} \%$ acetonitrile and $20 \mathrm{vol} \%$ D.I. water with 0.05 vol\% TFA. Linear gradient for competitive binding tests is from 65 to $10 \% \mathrm{~A}$ in $10 \mathrm{~min}$, and for real chicken white sample is from 100 to $0 \% \mathrm{~A}$ in $35 \mathrm{~min}$, with UV detection wavelength at $280 \mathrm{~nm}$, flow rate $0.8 \mathrm{~mL} / \mathrm{min}$ and injection volume $10 \mathrm{ul}{ }^{[5]}$.

\section{Results and Discussion}

\section{Binding properties of imprinted polymers}

Binding kinetics is a method to study the relationship between the absorb time and the binding capacity of MIPs(NIPs).

It is clear show in fig. 1 that the capacity of the MIPS(NIPs) keeps increasing with the time, and when the time reached some point, the speed of capacity increasing became slowly until it got adsorption equilibrium after 5 hours. At the beginning of the binding kinetics, a large amount of the high affinity imprinted locus are available in the imprinted polymers which make the target molecule can be easily absorbed with less resistance.

Lyz rapidly adsorbed on the MIP imprinted loci, the adsorption rate is larger than the desorption rate. So the adsorption capacity is growing rapidly; for NIPs, non-specific adsorption due to van der Waals forces and physical adsorption makes the adsorption capacity increase with the time growth, but the adsorption rate and adsorption capacity are less than MIPs. With the increasing of adsorption time, the adsorption capacity increases, and the imprinted polymers site gradually approaching saturation. When the template molecules lysozyme meet enough resistances during the process of entering the polymers, the adsorption rate decreases. While the rate of the desorption increases due to the protein molecule agglomeration. All those make the total polymer exhibits adsorption rate decreases, and gradually become balanced. MIPs reach the maximum amount of adsorption $3.7 \mathrm{mg} /$ $\mathrm{g}$ after 4 hours, and NIPs maximum adsorption capacity is $0.84 \mathrm{mg} / \mathrm{g}$. The Imprinted factor (IF) is 
4.4. Although the amount of polymer adsorption is not high, but the Imprinted factor is large, which indicates the polymer has a good adsorption selectivity for the template molecule lysozyme.

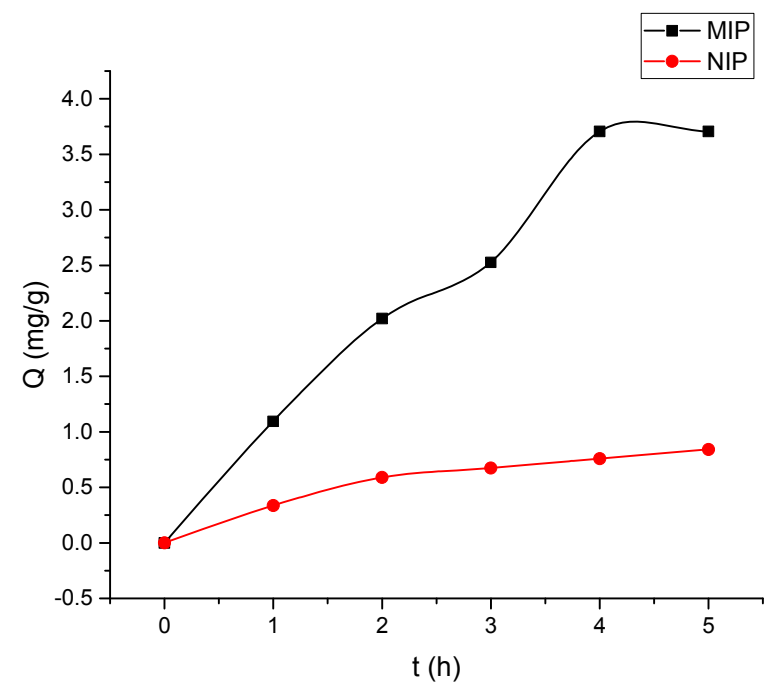

Fig.1Binding kinetics of MIPs and NIPs

Protein itself is with multiple charges, but under different $\mathrm{pH}$ conditions, the numbers of charges that proteinbrings are different. The number of charges that proteins bring would affect the interacting between proteins and functional monomers, and would affect MIPs adsorption capacity. Therefore, after determining the adsorption time, we also need to examine the affection of MIPs adsorption capacity due to the adsorption solution's $\mathrm{pH}$ changing. Since the template molecule's isoelectric point that experiments selected lysozyme is 11.0 , when the environment is below $\mathrm{pH}$ 11.0, lysozyme protonated positively charged, and the solution with $\mathrm{pH}$ change above it carboxyl and amino It will be different from the degree. Thus, if $\mathrm{pH}$ of the solution is too small, the adsorption process will be unfavorable, and too low $\mathrm{pH}$ will cause denaturation of lysozyme. In summary, the present study focuses on the changes of adsorption capacity of the MIPs when conditions' $\mathrm{pH}$ changes at 6.0-8.0. Formulated at $\mathrm{pH} 6.0,7.0$ and 8.0, at a concentration of lysozyme solution $250 \mu \mathrm{g} / \mathrm{mL}$ of adsorption experiments, five hours after adsorption, supernatant were detected after polymer adsorption with ultraviolet spectrophotometer, and the adsorption experimental results expressed in Figure 2.

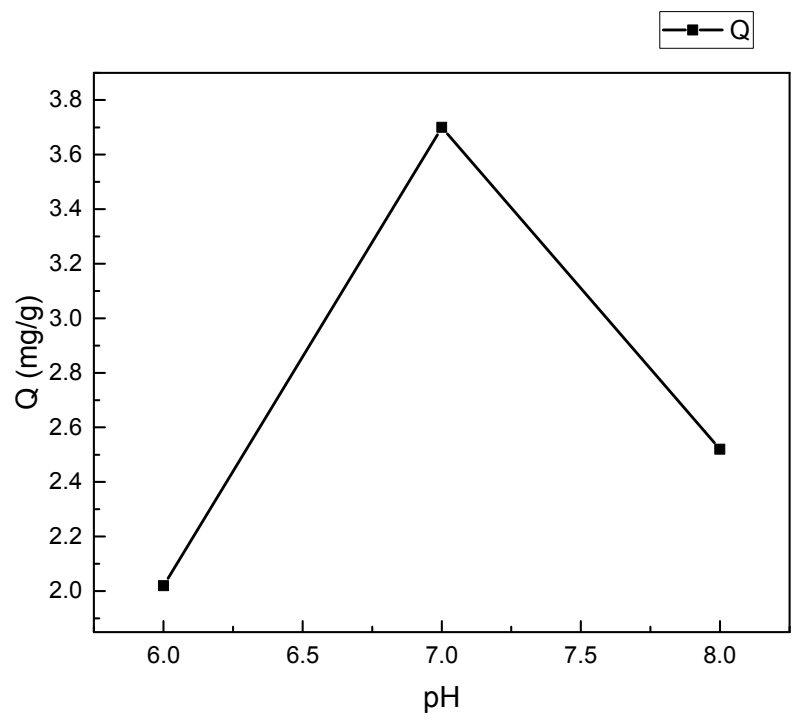

Fig 2 MIPs capacity with different $\mathrm{pH}$ 


\section{Binding isotherms}

In order to study adsorption properties of MIP and NIP with different concentration of template molecules lysozyme, at room temperature, choosing lysozyme concentrations were $0.05 \mathrm{mg} / \mathrm{ml} \sim$ $1.0 \mathrm{mg} / \mathrm{ml}$ (PBS, pH7.0) conditions doing the adsorption experiment. The experimental results are shown in Figure 3

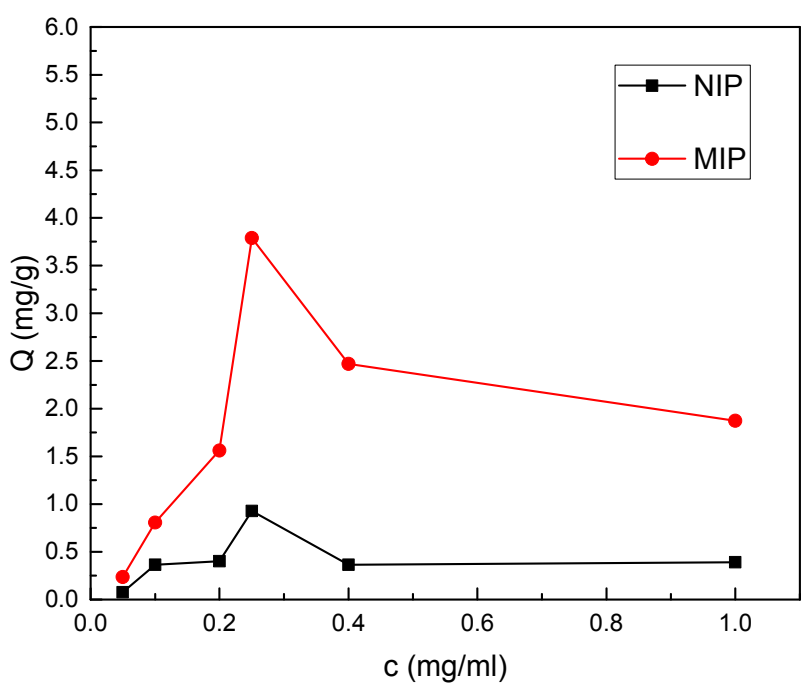

Fig.3Binding isotherm of MIPs andNIPs

As can be seen from Figure 3, MIPs' adsorption capacities for Lyz increase with increasing lysozyme concentration. When the lysozyme concentration was $0.25 \mathrm{mg} / \mathrm{ml}$, the binding sites on the MIPs got saturated and the adsorption process reached equilibrium. MIPs' maximum adsorption capacity is $3.78 \mathrm{mg} / \mathrm{g}$, and NIPs' maximum adsorption capacity is $0.92 \mathrm{mg} / \mathrm{g}$.

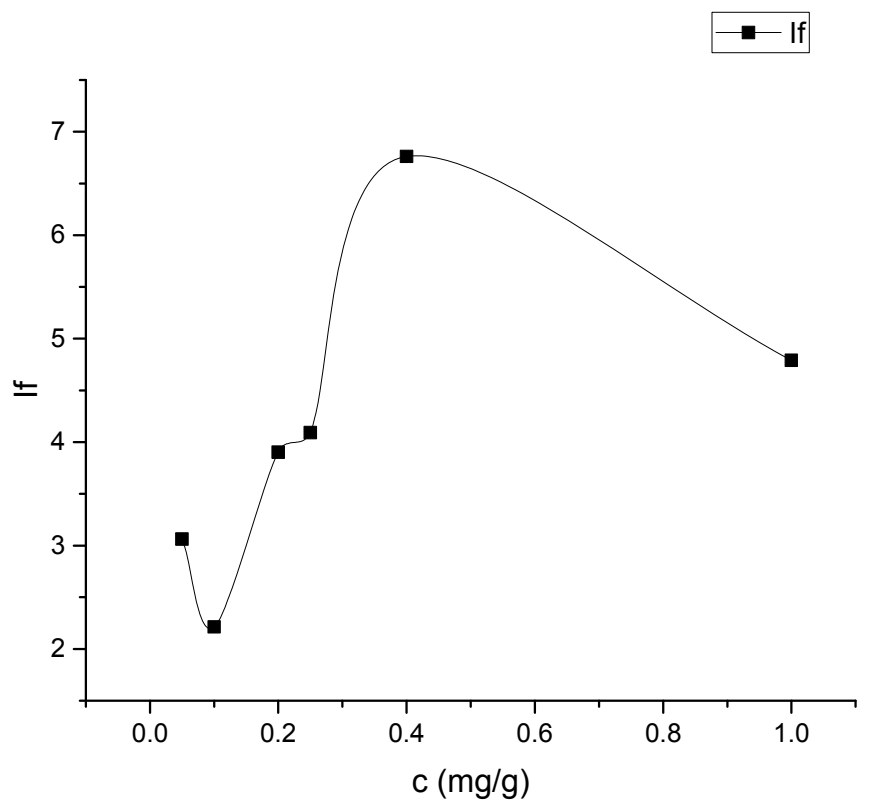

Fig.4Lysozyme concentration on the molecular imprinting factor(If)

From adsorption capacity data and imprint FACTOR (Figure 4) it can be seen, when the concentration of Lyz below $0.2 \mathrm{mg} / \mathrm{mL}$, the polymer adsorption data is not stable, it may be due to the low concentration of Lysozyme and operation error. When the concentration of lysozyme are in the $0.2 \mathrm{mg} / \mathrm{mL} \sim 1.0 \mathrm{mg} / \mathrm{mL}$ range, MIP can get good adsorption and stable data 


\section{Competitive binding test}

Table 1 shows different proteins of the peak area that the MIPs and NIPs adsorbed hybrid protein solution Lyz, BSA, OVA. Based on the peak area changes that molecularly imprinted polymers adsorbed protein in the solution before and after, it can reflect molecularly imprinted polymer membrane adsorption capacity for the three proteins.

Table 1 Before and after adsorption peak areas changes for different proteins

\begin{tabular}{|l|l|l|l|}
\hline Sample & Peak area (Lyz) & Peak area (BSA) & Peak area (OVA) \\
\hline hybrid protein solution & 547661 & 132257 & 62131 \\
\hline MIPs solution & 125276 & 107501 & 42743 \\
\hline NIPS solution & 395641 & 118445 & 54724 \\
\hline
\end{tabular}

\section{Conclusion.}

This study successfully imprinted Lysozyme polymers bybulk polymerization. The adsorption capacity is $3.7 \mathrm{mg} / \mathrm{g}$ and the Imprinted Factor is 6.7 . Even though the adsorption capacity is not very large, but the Imprinted Factor is really good. This indicates that the polymers have good selectivity and especially adsorbed the Lysozyme.

\section{References}

[1] $\mathrm{He} \mathrm{H,} \mathrm{Fu} \mathrm{G,} \mathrm{Wang} \mathrm{Y,} \mathrm{et} \mathrm{al.} \mathrm{Imprinting} \mathrm{of} \mathrm{protein} \mathrm{over} \mathrm{silica} \mathrm{nanoparticles} \mathrm{via} \mathrm{surface} \mathrm{graft}$ copolymerization using low monomer concentration[J]. Biosensors and Bioelectronics, 2010, 26(2): 760-765

[2] Hu Y L, Li Y W, Liu RJ,et al. Magnetic Molecularly Imprinted Polymer Beads Prepared by Microwave Heating forSelective Enrichment of $\beta$-Agonists in Pork and Pig Liver Samples[J].Talanta, 2011, 84(2): 462-470

[2] Lee M H, Chen Y C, Ho M H, et al. Optical recognition of salivary proteins by use of molecularly imprinted poly (ethylene-co-vinyl alcohol)/quantum dot composite nanoparticles[J]. Analytical and bioanalytical chemistry, 2010, 397(4): 1457-1466.

[3] Li D Y, Qin Y P, Li H Y, et al. A "turn-on" fluorescent receptor for detecting tyrosine phosphopeptide using the surface imprinting procedure and the epitope approach[J]. Biosensors and Bioelectronics, 2015, 66: 224-230.

[4] Liu J, Sun Z, Deng Y, et al. Highly Water - Dispersible Biocompatible Magnetite Particles with Low Cytotoxicity Stabilized by Citrate Groups[J]. AngewandteChemie, 2009, 121(32): 5989-5993.

[5]Gkementzoglou C, Kotrotsiou O, Kiparissides C (2013) Synthesisof novel compositenembranes based on molecularly imprintedpolymers for removal of triazine herbicides from water. IndEngChem Res 52:14001-1401 